\title{
Exploring Personalization of Gamification in an Introductory Programming Course
}

\section{Rogers, Mitchell}

ACM

2021-03-03

Rogers , M , Yao , W , Luxton-Reilly , A, Leinonen , J , Lottridge , D \& Denny , P 2021 , Exploring Personalization of Gamification in an Introductory Programming Course . in SIGCSE '21: Proceedings of the 52nd ACM Technical Symposium on Computer Science Education . ACM , pp. 1121-1127, ACM Technical Symposium on Computer Science Education , 13/03/2021 . https://doi.org/10.1145/3408877.3432402

http://hdl.handle.net/10138/328289

https://doi.org/10.1145/3408877.3432402

acceptedVersion

Downloaded from Helda, University of Helsinki institutional repository.

This is an electronic reprint of the original article.

This reprint may differ from the original in pagination and typographic detail.

Please cite the original version. 


\section{Exploring Personalization of Gamification in an Introductory Programming Course}

\author{
Mitchell Rogers \\ University of Auckland \\ Auckland, New Zealand \\ mrog173@aucklanduni.ac.nz \\ Juho Leinonen \\ University of Helsinki \\ Helsinki, Finland \\ juho.leinonen@helsinki.fi
}

\author{
Wendy Yao \\ University of Auckland \\ Auckland, New Zealand \\ wyao332@aucklanduni.ac.nz \\ Danielle Lottridge \\ University of Auckland \\ Auckland, New Zealand \\ d.lottridge@auckland.ac.nz
}

\author{
Andrew Luxton-Reilly \\ University of Auckland \\ Auckland, New Zealand \\ a.luxton-reilly@auckland.ac.nz \\ Paul Denny
University of Auckland
Auckland, New Zealand
paul@cs.auckland.ac.nz
}

\begin{abstract}
Gamification has been used in introductory programming courses, for example, to increase engagement with study materials, reduce procrastination, and increase attendance to practice sessions. Indeed, with the rapidly growing adoption of digital tools in such courses, the use of various game elements and mechanics to drive participation is increasing. Previous studies on gamification in computing have examined the effects over the whole student population. Prior work in other disciplines has found that the benefits associated with gamification may only be realized for some students, while others may even experience reduced motivation. The Hexad user types survey attempts to tackle this problem by grouping users into six different types for whom gamification should have different effects. The goal is to personalize the game elements for different user types, thus creating gamified experiences more suitable for individual learners. In this work, we study whether the Hexad survey could be used to guide the personalization of gamification in an introductory programming course. Specifically, we examine the quality of students' answers to the Hexad survey and explore whether they can be used to predict students' preferences for enabling gamification in the platform where they complete assignments. In our specific computing education context, we find that classifying students using the Hexad survey does not appear to be an effective approach for the automatic personalization of gamification.
\end{abstract}

\section{CCS CONCEPTS}

- Social and professional topics $\rightarrow$ Computing education

\section{KEYWORDS}

gamification, user types, hexad, personalization, programming

Permission to make digital or hard copies of all or part of this work for personal or classroom use is granted without fee provided that copies are not made or distributed for profit or commercial advantage and that copies bear this notice and the full citation on the first page. Copyrights for components of this work owned by others than the author(s) must be honored. Abstracting with credit is permitted. To copy otherwise, or republish, to post on servers or to redistribute to lists, requires prior specific permission and/or a fee. Request permissions from permissions@acm.org.

SIGCSE '21, March 13-20, 2021, Virtual Event, USA

(C) 2021 Copyright held by the owner/author(s). Publication rights licensed to ACM. ACM ISBN 978-1-4503-8062-1/21/03 ..\$15.00

https://doi.org/10.1145/3408877.3432402
ACM Reference Format:

Mitchell Rogers, Wendy Yao, Andrew Luxton-Reilly, Juho Leinonen, Danielle Lottridge, and Paul Denny. 2021. Exploring Personalization of Gamification in an Introductory Programming Course. In Proceedings of the 52nd ACM Technical Symposium on Computer Science Education (SIGCSE '21), March 13-20, 2021, Virtual Event, USA. ACM, New York, NY, USA, 7 pages. https://doi.org/10.1145/3408877.3432402

\section{INTRODUCTION}

Gamification is a broad term used to describe the integration of game-like mechanics and motivational affordances into non-game tasks to make them more engaging and enjoyable $[10,11]$. In the context of education, common motivational affordances include points, leaderboards, badges (achievements), levels, or challenges and these are typically designed to encourage positive study behaviors (or discourage negative ones) $[16,18]$. In computing education specifically, where digital learning tools are commonplace, the use of gamification is growing. Recent work has investigated the use of gamification to improve engagement with homework activities in a data structures course [12], to encourage task completion in an introductory programming course using a mastery learning approach [4], to reward positive study habits like starting work early in a CS1 course [32], to improve student time management on programming assignments [19] and to increase attendance at physical laboratory sessions [17].

Many applications implement gamification and measure its effectiveness in one context, but few studies look at the general issue of why gamification works, or why it does not work equally well for all users [24, 29]. In education, these are essential questions. A one-size-fits all model, where all students are presented the same motivational affordances, may not be the ideal way of motivating desired behaviors. Indeed, there is growing evidence from research in other disciplines that personalizing the gamification experience can lead to improved outcomes overall $[2,20,21]$. There are two general approaches to implementing personalization of gamification [35]. The first is customization, where the user deliberately selects the specific game elements they would like to have enabled. The second is adaptation, where the system determines the appropriate elements for the user automatically, perhaps informed by some other user-specific input such as personality information. Which of these two approaches is the most appropriate for students in computing courses is an open question. 
One promising method for adaptation is the Gamification User Types Hexad Scale, a survey created by Tondello et al. designed to assess an individual's user type [36]. The authors propose that the classified user type for an individual can then be used to determine the most appropriate game elements for them. In addition, they claim that this approach is more effective than customization where users are asked for their preferred elements directly - as the survey is derived from research on human motivation and personality.

In this research, we explore the feasibility of using the Hexad survey to personalize the gamification experience for students in an introductory programming course. Students interact with a gamified online tool that hosts questions they can answer for practice. The tool includes a set of achievement badges and a points-system with an associated leaderboard which are used to reward students for engaging with the practice activity. In addition to responding to the Hexad survey, we have students directly select their gamification preferences, and explore how these choices relate to the user types classification produced by the survey. To our knowledge, this is the first empirical work exploring use of the Hexad survey for personalizing gamification within an educational tool used in an authentic classroom setting.

We organize this work around the following two research questions:

- RQ1. In an educational context, are students' responses to the Hexad survey sufficiently high quality for classification into Hexad user types, and how does the distribution of types in this context compare to prior work?

- RQ2. To what extent do the Hexad classifications relate to students' explicit game element preferences?

\section{RELATED WORK}

\subsection{Gamification in Computing Education}

In computing education, gamification has been used within many educational tools to promote positive student behaviors. Dicheva et al. [12] found that gamification using badges and virtual currency within a Data Structures course caused students to practice significantly more compared to an earlier version of the course without the gamified elements. De Pontes et al. [4] conducted a randomized controlled trial in an introductory programming course where one group used a gamified platform with badges, a weekly leaderboard and personal record-tracking whereas the other group completed assignments without gamification. They found that students exposed to the gamification elements completed significantly more assignments over most weeks of the course. Hakulinen et al. [15] used achievement badges to motivate students to complete algorithm simulation exercises and to improve their study practices. They found that the gamified group was more likely to modify their behavior in order to obtain these badges, mirroring empirical results from other disciplines [5]. O'Donovan et al. [30] created a full gamification platform with a theme, levels, story, and skills to try to increase attendance at lectures and overall understanding in a tertiary game development course. In this study, both course grades and lecture attendance improved.
In addition to using gamification to have students practice more, gamification has also been used in introductory computer science classes to improve students' time management. Irwin and Edwards [19] introduced gamified elements common in mobile gaming into an introductory programming course. More specifically, students had a limited amount of "submission energy" which was needed for submitting work and which regenerated at the rate of one submission per hour. The goal of the study was to encourage students to start their work early and engage frequently with the assignment, as higher levels of procrastination tend to lead to lower quality outputs in computing courses $[8,14]$. They found that when compared to students in previous course iterations, students in the gamified version had improved scores and started work earlier, although both effects were small. Similarly, Sprint and Fox [32] found that students in a gamified course returned assignments earlier compared to a prior course without the gamified elements. Harrington and Chaudhry [17] found that giving students points that were visible in a course-wide leaderboard drastically increased participation in practice sessions compared to earlier course versions.

\subsection{Individual Differences in Gamification}

In gamification research, the common consensus is that gamification does work, but with some caveats [16]. A recurring theme in the literature is that gamification works only in specific environments, and only with certain users $[9,15]$. In most cases, only a small group of students are significantly affected by gamification. In their study involving programming students, De Pontes et al. [4] note that although overall there were significant differences between gamification and control groups, "the gamified platform had a more significant impact on some students than on others". One explanation for this is that different users have different behaviors and motivations when interacting with a gameful environment.

The classification of users into different user types is an active area of research in game environments, but is less studied with respect to gamification. One of the fundamental theories of gamer personalities was developed by Bartle [1]. He found that users interacting in a multi-user dungeon tended to have a preference for one of four playing styles: Achievers, Explorers, Killers, and Socialisers. Each of these four groups were attracted to different aspects of the game and gravitated towards a specific play style. Related to this, Tuunanen and Hamari [37] suggest that games designed with a specific player type in mind, by using elements appealing to that player type, result in that player type being dominant amongst the population of users.

The BrainHex model from Nacke et al. expands on the work of Bartle and others and presents a more modern approach to classifying players into archetypes [27, 28]. This model has been generalized to be more applicable to a wider variety of video games and takes inspiration from neurobiological research. Still, such models for personalities in video games do not directly apply to gameful experiences. For example, a student's motivation for using a gamified learning platform is not the same as their motivation for playing MMOs or other kinds of video games [36]. The Hexad model, originally created by Marczewski [25], is a recent and novel approach for quantifying differences between users. The six Hexad user types differ by the degree to which they are motivated by extrinsic or 
intrinsic factors. Classification of users into the Hexad user types can be achieved through the Hexad user types survey - a 24 question Likert scale survey to measure user preference for different elements in a gameful environment. The six Hexad user types as outlined by Tondello et al. [36] are:

(1) Achiever: Motivated by the need for competence, and strive to prove themselves by completing tasks.

(2) Disruptor: Motivated by triggering change and tend to disrupt the system.

(3) Free Spirit: Motivated by the need for autonomy and want the freedom to express themselves without external control

(4) Player: Motivated by extrinsic rewards, and will go out of their way to obtain these rewards.

(5) Philanthropist: Motivated by purpose and are willing to give without any reward.

(6) Socialiser: Motivated by relatedness and try to create social connections.

The user types in the Hexad model also draw inspiration from the Self Determination Theory (SDT) of Ryan and Deci [31]. SDT is commonly used to explain why gamification works [3, 9, 33] Ryan and Deci hypothesize that humans have three characteristic psychological needs, for competence, autonomy, and relatedness [31] From these three intrinsic needs, four of the Hexad user types are derived: Socialisers, Philanthropists, Achievers, and Free Spirits.

\section{METHODS}

To address our research questions, we conducted an experiment involving 1026 first-year undergraduate engineering students taking an introductory programming course at the University of Auckland. These students interacted with an online study tool, PeerWise [7], which hosted questions that could be answered for practice and exam preparation [6]. Students earned $2 \%$ towards their final course grade for generating a minimum of three and answering a minimum of six practice questions prior to the course exam. Authoring and answering more than these minimum targets was allowed, and the game elements in PeerWise were designed to encourage this. For example, students would earn points for every correctly answered question (and lose points for incorrect answers), and the top pointscorers were listed on a leaderboard table. Badges were awarded to students for achieving answering targets, such as answering 10 questions correctly in a row, or answering 10 questions on each of five consecutive days. All such participation, over and above the minimum target for earning the $2 \%$ course credit, was optional and ungraded.

The data we report here, in which we examine students' Hexad classifications and gamification preferences, was collected as part of a larger experiment in which all 1026 students were randomly assigned to one of three groups: a "control" group, a "gamification" group, and a "choice" group (see Table 1). For the control group, none of the gamification elements were visible in the user interface. For the gamification group, all gamification elements (badges, points and leaderboards) were visible. For the choice group, students had the choice to enable (show) or disable (hide) any of the game elements. Specifically, the choices were to view 'No game elements', 'Points and leaderboards only', 'Badges only', or 'All game elements'.
Table 1: Summary of group sizes (random assignment)

\begin{tabular}{c|ccc|c}
\hline & Control & Gamification & Choice & Total \\
\hline Students & 339 & 344 & 343 & 1026 \\
\hline
\end{tabular}

The 24-question 7-response Likert scale Hexad survey proposed by Tondello et al. [36] was presented to all students upon their first login to PeerWise. The questions on this survey include four questions for each of the six Hexad user types. We recorded the time at which the Hexad survey was displayed to students, and the time at which the survey was submitted. From this, we compute the time elapsed for responding to the survey. Of the 1026 students enrolled in the course, 1016 completed the Hexad survey and interacted with PeerWise.

Pertinent to this experiment are the Achiever and Player user types. These are the two types hypothesized to be motivated by the kinds of rewards available in our gamified tool: points, badges, and leaderboards. These two user types differ with Achievers focusing on intrinsic competence and Players focusing on extrinsic rewards [36]. Achievers are likely motivated by the challenges inherent in the platform, such as answering questions correctly, targeting their need for competence. We expect the students in the Player user type group, who are hypothesized to be strongly motivated by extrinsic rewards, to be most likely to enable all gamification elements when given the choice.

\section{RESULTS}

\subsection{Quality of Hexad survey responses}

To answer our first research question, we assessed the quality of the responses to the Hexad survey by examining the variation in responses for a given student as well as timestamped logs of behavior.

We examined response variation to understand if students appeared to submit thoughtful responses. The mode response for a submitted survey is the quantitative Likert level (e.g. "agree") that is most often selected. For a given survey, the maximum number of times the mode response can be selected is 24 (if every item on the survey is answered with the mode response). Figure 1 plots a histogram showing the number of times the mode response occurred across all surveys. The peak on the far right, at 24 on the horizontal axis, represents the 137 students who selected the same response for all $24 \mathrm{Hexad}$ questions. In such cases, where there is no variation in responses, we consider the responses to be low quality (for example, they may indicate that a student has filled down one column of the survey instrument without thinking carefully about their answers). A smaller proportion of students responded to all but a few of the questions with an identical response. We wish to exclude low quality responses from our later analysis of Hexad types. For the purposes of this analysis, we exclude students if the overall variance (when the Likert items are numerically coded) across their responses to the Hexad questions is less than 0.7 .

We also assessed response quality by looking at the time taken to complete the survey. In particular, we were interested in identifying students who completed the survey in less time than is reasonable to simply read the survey text. Plotting the time taken by all students 


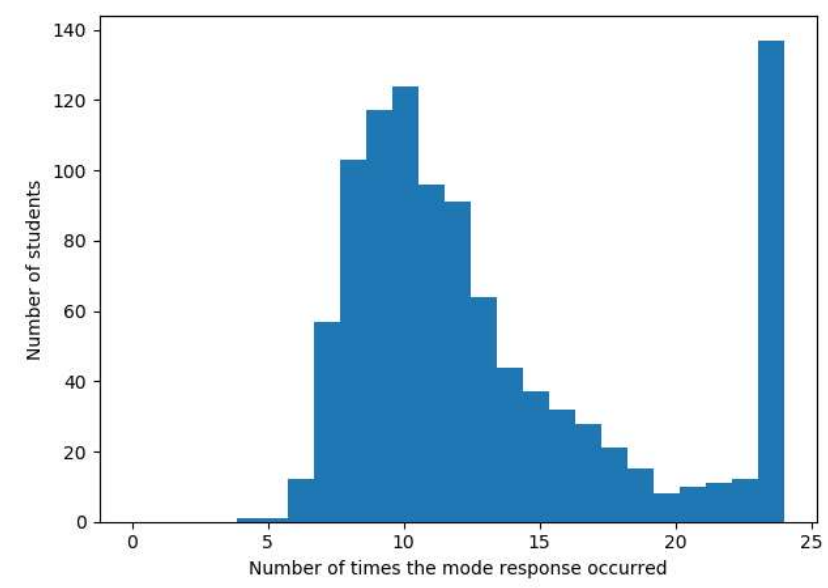

Figure 1: Frequency distribution of the number of times the mode response, or most commonly selected Likert level, occurred across all surveys.

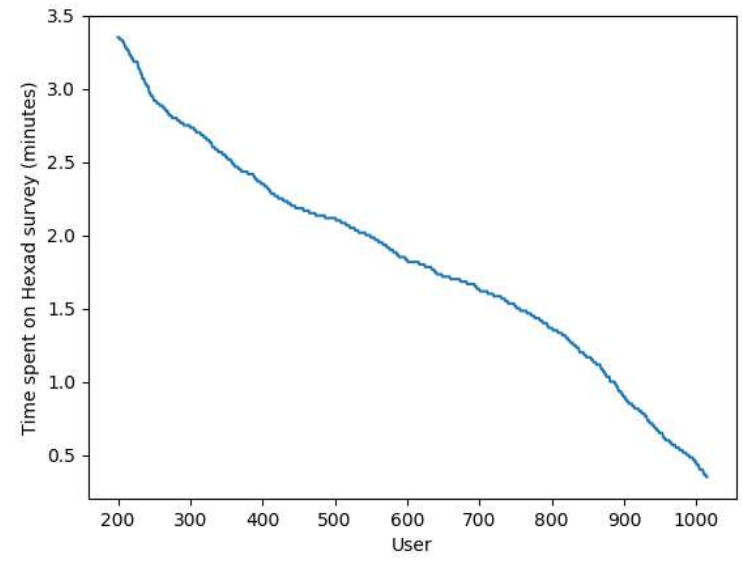

Figure 2: Time spent on Hexad survey, sorted, for fastest 816 students (slowest 200 not shown).

to complete the survey resulted in a logarithmic distribution, with a small number of students exhibiting a large elapsed survey time, with a maximum of 69 minutes and a minimum of 21 seconds. The median time taken to complete the survey was a little over 2 minutes, and 89 students spent longer than 5 minutes on the survey. Figure 2 shows the time spent on the survey by 816 students sorted in decreasing order - note this data excludes the 200 slowest students which would otherwise result in a very flat plot.

The total length of the 24-items on the Hexad survey is 189 words. Combined with the instructions on the questionnaire page, totalling 46 words, our participants were reading around 235 words as well as selecting responses on the 7-point Likert scale for each item. Dyson and Haselgrove explored comprehension rates when participants read from a screen, and found that a normal rate of

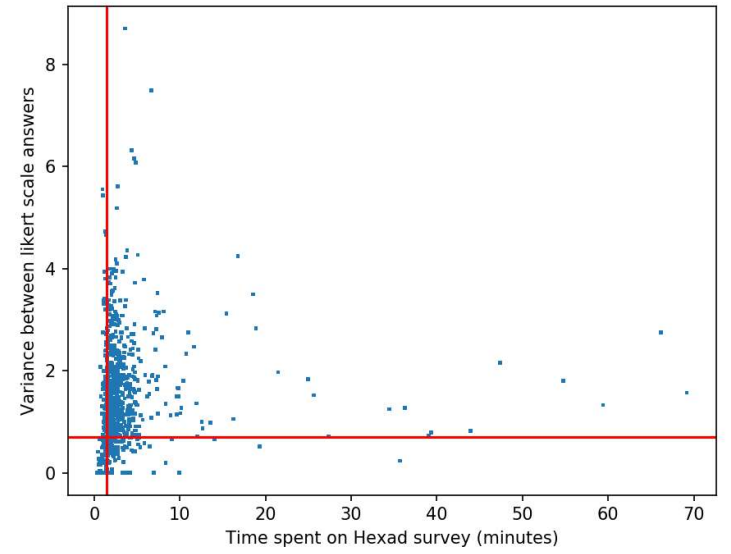

Figure 3: Time spent on Hexad survey plotted against variance in selected responses, for all students. Red lines indicate thresholds used for data cleaning.

reading was 151 words per minute [13]. A reasonable estimate then, for simply reading the text of the survey without thinking about or entering responses, is around one and a half minutes. We use this time as a threshold - that is, for our later analysis we exclude those students who responded to all questions on the 24-item survey in less than one and a half minutes.

Figure 3 plots the response variance against the time spent on the survey for all students, and overlays red lines indicating the thresholds below which we excluded students from our later analysis. The vertical line is at the 90 second mark (1.5 minutes), and the horizontal line is at a variance across responses of 0.7 . This data cleaning step reduced the sample of 1016 students to 638 students.

\subsection{Hexad user types and explicit choices}

When given a choice regarding gamification (i.e. all students assigned to the "choice" group), we observed that students tended to either enable or disable all game elements, rather than opting to make only one kind of element visible. Out of the 343 students initially assigned to the "choice" group, 336 participated. Of these, 107 chose to be shown no game elements, 12 chose only points, 13 chose only badges, and 204 chose all game elements. Students' choices separated by user type are shown in Table 2.

In addition, although students in the "choice" group had the option of changing their game element selections, very few did Only 18 students switched groups after their initial selection. There was a total of 25 switches throughout the study period, and over half of these switches were students choosing to see all of the game elements after initially selecting to disable them. Students' choices over the duration of the course are visualized in Figure 4.

Each student's disposition towards each user type was determined by summing the responses for each group of four questions applicable to the user type, as described in Tondello et al. [36]. The primary user type for each student was determined by taking the user type with the highest score out of the six user types. Out of the 


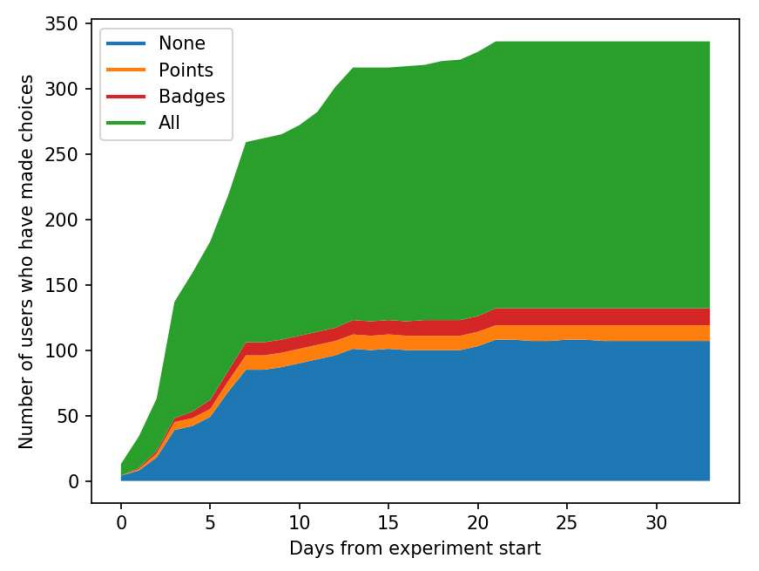

Figure 4: Distribution of gamification selections, for students in the "choice" group, over experiment timeframe.

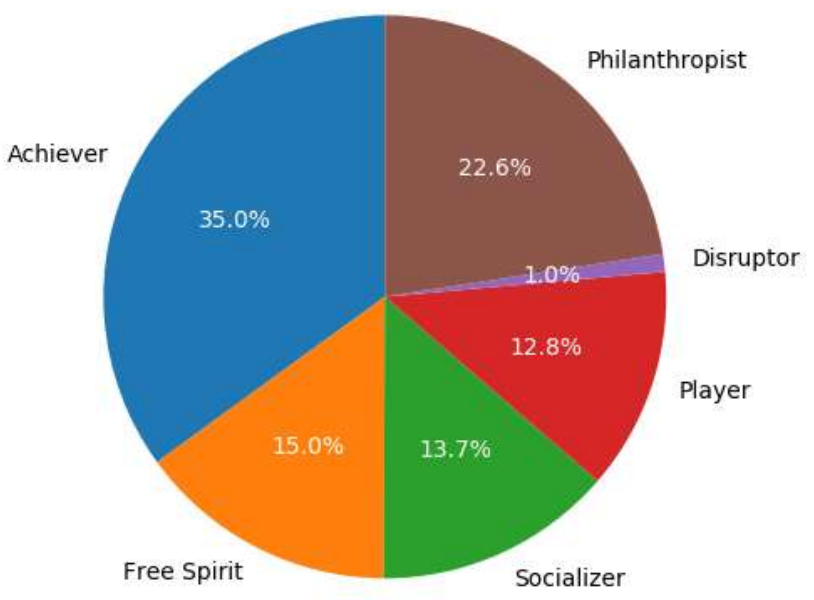

Figure 5: Distribution of Hexad user types.

638 students remaining after the data cleaning described in Section 4.1, 140 students had a two-way tie for their primary user type. Additionally, 35 students had a three-way tie for their primary user type, and 12 students had either a four or five-way tie. In cases where students had a tie for their primary user type, we discarded students with a four or five-way tie. Tondello et al. [34, 36] do not outline what to do in this scenario. To plot the distribution of user types, we weighted each user type by a third for three-way ties and a half for two-way ties to count students, i.e. a student who was an Achiever and a Player would count as half a student towards each of these. The distribution of user types is shown in Figure 5 and is similar to what has been reported in previous studies, with a low number of Disruptors, and with the intrinsically motivated types being the most common.
Table 2: Summary of gamification selections, for students in the "choice" group, by user type with ties ignored.

\begin{tabular}{|c|cccc|}
\hline User type & None & Points & Badges & All \\
\hline Achiever & 17 & 4 & 3 & 36 \\
Disruptor & 1 & 0 & 0 & 1 \\
Free spirit & 3 & 3 & 3 & 16 \\
Philanthropist & 7 & 0 & 2 & 22 \\
Player & 7 & 0 & 0 & 9 \\
Socialiser & 7 & 0 & 0 & 11 \\
\hline Total & 42 & 7 & 8 & 95 \\
\hline
\end{tabular}

We now explore how well the classified user types relate to the students' explicit game element preferences. The Player user type is associated with extrinsic rewards, and elements such as points, leaderboards and badges are all suggested as effective motivators for this type [36]. We would therefore expect that students who are classified as Players would be more likely to enable the game elements than students in the other user type groups. We use a $\chi^{2}$ test to examine this relationship. The null hypothesis in this case is that each student's gamification choice (whether to view the game elements or not) is independent of that student's user type classification (whether a Player or not).

Since it is unclear how to determine the user type for students who were equally likely to be part of two or more groups, these students were excluded. Including only students with a single primary user type satisfies the assumptions of the $\chi^{2}$ test, since any student can only be counted in one row and one column. We collapse the columns of Table 2 into two categories, either: game elements ("Points", "Badges", and "All game elements") or no game elements. We also collapse the rows such that the Player type is compared against the other five user types.

The proportion of students who chose to enable game elements does not differ significantly between the two groupings of Hexad user types, $\chi^{2}(1, N=152)=2.3234, p=.1274$. Therefore, we fail to reject the null hypothesis - we do not have sufficient evidence that students' explicit gamification choices are significantly related to their Hexad user type classifications.

\section{DISCUSSION}

In this research, we explored the feasibility of using the Hexad survey to assign gamification elements to computing students in an introductory programming course. In answer to our first research question, we found that many students responded to the survey in ways that suggested their data may not be meaningful - completing the survey too quickly and often with little to no variation in responses. For those that did provide high quality responses, amounting to a little over half of the class, the distribution into the six Hexad types was roughly similar to distributions reported in contexts outside of computer science classrooms.

In answer to our second research question, we found no evidence that students classified into the Player user type were any more likely to enable the game elements than students in the other user types. The Player user type is the type that is most strongly associated with the kinds of game elements that students could 
select to see within the interface of PeerWise. We would expect that students associated with this user type would therefore have a stronger tendency to enable the game elements when given the choice. The fact that we didn't find such a relationship raises interesting questions around whether students are able to select effective game elements for themselves, or whether the Hexad survey is a practical instrument to use in this context. The benefits of adaptive personalization over customized gamification are only realized if students are unable to make accurate estimations of the game elements that work best for them.

We found that students tended to either disable the game elements altogether, or enable all of them, rather than selecting to see one set of elements and not the other. This behavior makes sense if we consider that some students are naturally driven by extrinsic rewards. If a student chooses to enable badges because they are motivated by extrinsic rewards, they are likely to also find that points and leaderboards act as effective extrinsic motivators. We also observed that very few students in the choice group changed their selections during the experiment. This lends some support to the idea that students are, in fact, able to make good decisions about the game elements that will suit them, simply by being given a direct choice. We saw little evidence that students were not satisfied with the initial choices they made.

In contrast to previous implementations of this survey in other contexts [36], we found a slightly different distribution of user types. This is interesting because the population of students taking this first-year programming course was more homogenous than the samples from previous surveys. The variation in age and background is smaller than in prior work by Tondello et al. [34]. Our local context may also explain why we see a higher proportion of Achievers in the distribution of user types. The course in which our experiment was conducted is a highly competitive course, in which course performance determines entry into specializations in later years, and students are therefore highly motivated by the need for competence [31]. In our context we also saw a lower proportion of Socialiser and Free Spirit user types. Tondello et al. [36] found that scores towards extrinsically motivated user types decrease with age and scores towards intrinsically motivated types such as Philanthropist and Socialiser tend to increase with age. Given that our participants were all in their first year of study, this may explain the relatively higher proportion of extrinsically motivated Player types. Future work exploring how these distributions differ across other academic disciplines, outside of computing and even outside of the sciences, would make a fascinating comparison with the data we present here.

In cleaning our data for analysis, we observed that a large proportion (around $40 \%$ ) of responses to the survey were deemed to be low quality. Similar issues with questionnaire answers [23] and selfreported metrics [22] have been found previously. That such a large proportion of students seemed unwilling to complete the Hexad survey suggests it may work best alongside other approaches to personalization. Offering participants a choice, between customizing their game elements through direct choice, or by completing a survey like the Hexad survey, may be a practical way to implement personalization. Certainly our study differs from previous studies by Tondello et al. [34, 35], in which no data quality issues were reported.

\section{THREATS TO VALIDITY}

\subsection{Extrinsic rewards}

Students meeting the requirements for creating and answering practice questions on PeerWise were rewarded with $2 \%$ towards their final grade in the course. This mark incentive is itself an extrinsic reward (many students may be engaging with the activity to earn these marks, rather than being intrinsically motivated by the practice activity itself). Although $2 \%$ is a relatively small weighting, the course is highly competitive and marks are a very effective motivator for many students. Students may have valued the marks they would earn from the activity much more highly than the rewards within the tool. In addition, the summative tests used in the course also reused some of the questions from PeerWise, introducing another external incentive for students to use the platform. Future work should explore this in an optional setting, where there is no compulsion for students to participate, and where the game elements may have a higher perceived importance.

\subsection{Biases towards certain user types}

A limitation of this work is that only a very limited set of game elements were explored. Other kinds of elements, such as quests, social discovery, and more exploratory tasks, are hypothesized to appeal to some of the other Hexad user types. Bartle also suggests that there are interactions between different player types [1] which also apply to the Hexad model. Socialisers need other users to be present in the platform to thrive, and Players need other users to compare themselves to. Students remain anonymous to one another within PeerWise, a design choice which has been shown to improve participation and reduce certain kinds of biases [26], but one which may also limit the effectiveness of elements such as the leaderboards.

\section{CONCLUSION}

Our study is the first to evaluate the use of the Hexad survey for personalizing gamification in a tertiary computing education context. In their previous work, Tondello et al. [34-36] found relationships between survey responses and users' preferred game elements, but their sample provided higher quality responses than our sample provided. Low quality responses may be a limiting factor of the Hexad model in education contexts. In our study, we found no evidence that students classified as the Player user type were more likely to prefer game elements. However, the relatively small variety of game elements we used limited our findings and meant we were unable to explore additional Hexad user types.

We found that most students either selected all game elements or no game elements. This pattern is in line with Marczewski's [25] view of the different user types: all three game elements available in our experiment were extrinsic rewards targeting competence. We recommend that future research be conducted with a platform that includes a more extensive range of game elements. Our results arise from a sample of students in a tertiary computing course and future work in more diverse educational contexts may provide stronger evidence in favor of adaptation. Until such time, providing students with the ability to customize their own experience may be a more sound approach than automatic personalization. 


\section{REFERENCES}

[1] Richard Bartle. 1996. Hearts, clubs, diamonds, spades: Players who suit MUDs. fournal of MUD research 1, 1 (1996), 19

[2] Martin Böckle, Jasminko Novak, and Markus Bick. 2017. Towards Adaptive Gamification: A Synthesis of Current Developments. In Proc of the Twenty-Fifth European Conf on Information Systems (Guimaraes, Portugal) (ECIS '17). 158-174.

[3] Yang Chen. 2018. Understanding How Educational Gamification Impacts Users Behavior: A Theoretical Analysis. In Proceedings of the 6th International Conference on Information and Education Technology (Osaka, Japan) (ICIET '18) Association for Computing Machinery, New York, NY, USA, 154-159. https: //doi.org/10.1145/3178158.3178188

[4] Rafael G. de Pontes, Dalton D. S. Guerrero, and Jorge C. A. de Figueiredo. 2019. Analyzing Gamification Impact on a Mastery Learning Introductory Programming Course. In Proceedings of the 50th ACM Technical Symposium on Computer Science Education (Minneapolis, MN, USA) (SIGCSE '19). Association for Computing Machinery, New York, NY, USA, 400-406. https://doi.org/10.1145/3287324.3287367

[5] Paul Denny. 2013. The Effect of Virtual Achievements on Student Engagement In Proceedings of the SIGCHI Conference on Human Factors in Computing Systems (Paris, France) (CHI '13). Association for Computing Machinery, New York, NY, USA, 763-772. https://doi.org/10.1145/2470654.2470763

[6] Paul Denny. 2015. Generating Practice Questions as a Preparation Strategy for Introductory Programming Exams. In Proceedings of the 46th ACM Technical Symposium on Computer Science Education (Kansas City, Missouri, USA) (SIGCSE '15). Association for Computing Machinery, New York, NY, USA, 278-283. https: //doi.org/10.1145/2676723.2677253

[7] Paul Denny, John Hamer, Andrew Luxton-Reilly, and Helen Purchase. 2008. PeerWise: Students Sharing Their Multiple Choice Questions. In Proceedings of the Fourth International Workshop on Computing Education Research (Sydney, Australia) (ICER '08). Association for Computing Machinery, New York, NY, USA, 51-58. https://doi.org/10.1145/1404520.1404526

[8] Paul Denny, Andrew Luxton-Reilly, Michelle Craig, and Andrew Petersen. 2018. Improving Complex Task Performance Using a Sequence of Simple Practice Tasks. In Proc of the 23rd Annual ACM Conf on Innovation and Technology in Computer Science Education (Larnaca, Cyprus) (ITiCSE 2018). Association for Computing Machinery, New York, NY, USA, 4-9. https://doi.org/10.1145/3197091.3197141

[9] Paul Denny, Fiona McDonald, Ruth Empson, Philip Kelly, and Andrew Petersen. 2018. Empirical Support for a Causal Relationship Between Gamification and Learning Outcomes. In Proceedings of the 2018 CHI Conference on Human Factors in Computing Systems (Montreal QC, Canada) (CHI '18). Association for Computing Machinery, New York, NY, USA, 1-13. https://doi org/10.1145/31735743173885

[10] Sebastian Deterding, Dan Dixon, Rilla Khaled, and Lennart Nacke. 2011. From Game Design Elements to Gamefulness: Defining "Gamification". In Proceedings of the 15th International Academic MindTrek Conference: Envisioning Future Media Environments (Tampere, Finland) (MindTrek '11). Association for Computing Machinery, New York, NY, USA, 9-15. https://doi.org/10.1145/2181037.2181040

[11] Christo Dichev, Darina Dicheva, Galia Angelova, and Gennady Agre. 2015. From Gamification to Gameful Design and Gameful Experience in Learning. Cybern Inf. Technol. 14, 4 (Jan. 2015), 80-100. https://doi.org/10.1515/cait-2014-0007

[12] Darina Dicheva, Keith Irwin, and Christo Dichev. 2019. OneUp: Engaging Students in a Gamified Data Structures Course. In Proceedings of the 50th ACM Technical Symposium on Computer Science Education (Minneapolis, MN, USA) (SIGCSE '19). Association for Computing Machinery, New York, NY, USA, 386-392. https://doi.org/10.1145/3287324.3287480

[13] Mary Dyson and Mark Haselgrove. 2001. The influence of reading speed and line length on the effectiveness of reading from screen. Int. 7. Human-Computer Studies 54 (2001), 585-612

[14] Stephen H. Edwards, Jason Snyder, Manuel A. Pérez-Quiñones, Anthony Allevato, Dongkwan Kim, and Betsy Tretola. 2009. Comparing Effective and Ineffective Behaviors of Student Programmers. In Proceedings of the Fifth International Workshop on Computing Education Research Workshop (Berkeley, CA, USA) (ICER '09). Association for Computing Machinery, New York, NY, USA, 3-14. https://doi.org/10.1145/1584322.1584325

[15] Lasse Hakulinen, Tapio Auvinen, and Ari Korhonen. 2013. Empirical Study on the Effect of Achievement Badges in TRAKLA2 Online Learning Environment In Proc of the 2013 Learning and Teaching in Computing and Engineering (LATICE '13). IEEE Computer Society, USA, 47-54. https://doi.org/10.1109/LaTiCE.2013.34

[16] Juho Hamari, Jonna Koivisto, and Harri Sarsa. 2014. Does Gamification Work? A Literature Review of Empirical Studies on Gamification. In Proceedings of the 2014 47th Hawaii International Conference on System Sciences (HICSS '14). IEEE Computer Society, USA, 3025-3034. https://doi.org/10.1109/HICSS.2014.377

[17] Brian Harrington and Ayaan Chaudhry. 2017. TrAcademic: Improving Partic ipation and Engagement in CS1/CS2 with Gamified Practicals. In Proceedings of the 2017 ACM Conference on Innovation and Technology in Computer Science Education (Bologna, Italy) (ITiCSE '17). Association for Computing Machinery, New York, NY, USA, 347-352. https://doi.org/10.1145/3059009.3059052

[18] Theresia Devi Indriasari, Andrew Luxton-Reilly, and Paul Denny. 2020. Gamification of student peer review in education: A systematic literature review. $E d$. and Info. Tech. 25 (2020), 5205-5234. https://doi.org/10.1007/s10639-020-10228-x
[19] Michael S. Irwin and Stephen H. Edwards. 2019. Can Mobile Gaming Psychology Be Used to Improve Time Management on Programming Assignments?. In Proceedings of the ACM Conference on Global Computing Education (Chengdu, Sichuan, China) (CompEd '19). Association for Computing Machinery, New York, NY, USA, 208-214. https://doi.org/10.1145/3300115.3309517

[20] Ana Klock, Marcelo Pimenta, and Isabela Gasparini. 2018. A Systematic Mapping of the Customization of Game Elements in Gamified Systems. In Proceedings of SBGames 2018 (Foz do Iguacu, Brazil) (SBGames '18). 11-18.

[21] Ana Carolina Tomé Klock, Isabela Gasparini, Marcelo Soares Pimenta, and Juho Hamari. 2020. Tailored gamification: A review of literature. Int. F. of HumanComputer Studies 144 (2020), 102495. https://doi.org/10.1016/j.ijhcs.2020.102495

[22] Juho Leinonen, Leo Leppänen, Petri Ihantola, and Arto Hellas. 2017. Comparison of Time Metrics in Programming. In Proceedings of the 2017 ACM Conference on International Computing Education Research (Tacoma, Washington, USA) (ICER '17). Association for Computing Machinery, New York, NY, USA, 200-208. https //doi.org/10.1145/3105726.3106181

[23] Leo Leppänen, Juho Leinonen, and Arto Hellas. 2016. Pauses and Spacing in Learning to Program. In Proceedings of the 16th Koli Calling International Conference on Computing Education Research (Koli, Finland) (Koli Calling '16). Association for Computing Machinery, New York, NY, USA, 41-50. https: //doi.org/10.1145/2999541.2999549

[24] Pascal Lessel, Maximilian Altmeyer, Marc Müller, Christian Wolff, and Antonio Krüger. 2017. Measuring the Effect of "Bottom-up" Gamification in a Microtask Setting. In Proceedings of the 21st International Academic Mindtrek Conference (Tampere, Finland) (AcademicMindtrek '17). Association for Computing Machinery, New York, NY, USA, 63-72. https://doi.org/10.1145/3131085.3131086

[25] Andrzej Marczewski. 2015. User Types. In Even Ninja Monkeys Like to Play: Gamification, Game Thinking and Motivational Design (1st ed.). CreateSpace Independent Publishing Platform, 165- 177.

[26] Gabriela Morales-Martinez, Paul Latreille, and Paul Denny. 2020. Nationality and Gender Biases in Multicultural Online Learning Environments: The Effects of Anonymity. In Proceedings of the 2020 CHI Conference on Human Factors in Computing Systems (Honolulu, HI, USA) (CHI '20). Association for Computing Machinery, New York, NY, USA, 1-14. https://doi.org/10.1145/3313831.3376283

[27] Lennart Nacke, Chris Bateman, and Regan Mandryk. 2011. BrainHex: Preliminary Results from a Neurobiological Gamer Typology Survey, Vol. 6972. 288-293. https://doi.org/10.1007/978-3-642-24500-8 31

[28] Lennart E. Nacke, Chris Bateman, and Regan L. Mandryk. 2014. BrainHex: A neurobiological gamer typology survey. Entertainment Computing 5, 1 (2014), 55 - 62. https://doi.org/10.1016/j.entcom.2013.06.002

[29] Rita Orji, Julita Vassileva, and Regan L. Mandryk. 2014. Modeling the Efficacy of Persuasive Strategies for Different Gamer Types in Serious Games for Health User Modeling and User-Adapted Interaction 24, 5 (Dec. 2014), 453-498. https: //doi.org/10.1007/s11257-014-9149-8

[30] Siobhan O'Donovan, James Gain, and Patrick Marais. 2013. A Case Study in the Gamification of a University-Level Games Development Course. In Proceedings of the South African Institute for Computer Scientists and Informa tion Technologists Conference (East London, South Africa) (SAICSIT '13). Association for Computing Machinery, New York, NY, USA, 242-251. https //doi.org/10.1145/2513456.2513469

[31] Richard Ryan and Edward Deci. 2000. Self-Determination Theory and the Facilitation of Intrinsic Motivation, Social Development, and Well-Being. The American psychologist 55 (2000), 68-78. https://doi.org/10.1037/0003-066X.55.1.68

[32] Gina Sprint and Erik Fox. 2020. Improving Student Study Choices in CS1 with Gamification and Flipped Classrooms. In Proceedings of the 51st ACM Technical Symposium on Computer Science Education (Portland, OR, USA) (SIGCSE '20). Association for Computing Machinery, New York, NY, USA, 773-779. https: //doi.org/10.1145/3328778.3366888

[33] Sarah-Kristin Thiel. 2016. Reward-Based vs. Social Gamification: Exploring Effectiveness of Gamefulness in Public Participation. In Proceedings of the 9th Nordic Conference on Human-Computer Interaction (Gothenburg, Sweden) (NordiCHI '16). Association for Computing Machinery, New York, NY, USA, Article 104, 6 pages. https://doi.org/10.1145/2971485.2996739

[34] Gustavo F. Tondello, Alberto Mora, Andrzej Marczewski, and Lennart E. Nacke. 2019. Empirical validation of the Gamification User Types Hexad scale in English and Spanish. International fournal of Human-Computer Studies 127 (2019), 95 111. https://doi.org/10.1016/j.ijhcs.2018.10.002

[35] Gustavo F. Tondello and Lennart E. Nacke. 2020. Validation of User Preferences and Effects of Personalized Gamification on Task Performance. Frontiers in Computer Science 2 (2020), 1-23. https://doi.org/10.3389/fcomp.2020.00029

[36] Gustavo F. Tondello, Rina R. Wehbe, Lisa Diamond, Marc Busch, Andrzej Marczewski, and Lennart E. Nacke. 2016. The Gamification User Types Hexad Scale In Proceedings of the 2016 Annual Symposium on Computer-Human Interaction in Play (Austin, Texas, USA) (CHI PLAY '16). Association for Computing Machinery, New York, NY, USA, 229-243. https://doi.org/10.1145/2967934.2968082

[37] Janne Tuunanen and Juho Hamari. 2012. Meta-synthesis of player typologies. In DiGRA Nordic '12: Proceedings of 2012 International DiGRA Nordic Conference http://www.digra.org/wp-content/uploads/digital-library/12168.40312.pdf 